Bulletin of Mathematical Biology (1999) 61, 0-0

Article No. bulm.1999.0124

Available online at http://www.idealibrary.com on IDE

\title{
Erratum*
}

\section{Diffusively Coupled Bursters: Effects of Cell Heterogeneity}

\author{
GERDA DE VRIES, ARTHUR SHERMAN \\ Mathematical Research Branch, \\ NIDDK, National Institutes of Health, \\ Bethesda, MD 20892, \\ U.S.A. \\ E-mail: gerda@helix.nih.gov \\ E-mail: sherman@helix.nih.gov \\ HSIU-RONG ZHU \\ 16203 S. 26th Place, \\ Phoenix, AZ 85048, \\ U.S.A. \\ E-mail: zhu@jchslab.la.asu.edu
}

Bulletin of Mathematical Biology (1998) 60, 1167-1200.

The authors of the above paper would like to point out that in Fig. 8, the curve separating regions $\mathrm{C}$ and $\mathrm{D}$ in the right halfplane should be $\gamma_{A P}$, not $\gamma_{I P}$. Similarly, the curve separating regions A and B in the right halfplane is mislabelled.

* These errors have been corrected on the online (IDE $\mathbf{C}$ ) version of the article.

$0092-8240 / 99 / 050000+01 \quad \$ 30.00 / 0$

(C) 1999 Society for Mathematical Biology 